\title{
Effet d'une expérimentation de brassage artificiel epilimnique par aération sur les poussées cyanobactériennes dans la retenue hypereutrophe de Grangent (France)
}

\section{Impact of an epilimnic artificial mixing experiment against growth of cyanobacteria in the hypertrophic reservoir of Grangent (France)}

\author{
D. Latour-Duris, H. Giraudet et J.-L. Berthon
}

Volume 15, numéro 1, 2002

URI : https://id.erudit.org/iderudit/705453ar

DOI : https://doi.org/10.7202/705453ar

Aller au sommaire du numéro

Éditeur(s)

Université du Québec - INRS-Eau, Terre et Environnement (INRS-ETE)

ISSN

0992-7158 (imprimé)

1718-8598 (numérique)

Découvrir la revue

Citer cet article

Latour-Duris, D., Giraudet, H. \& Berthon, J.-L. (2002). Effet d'une expérimentation de brassage artificiel epilimnique par aération sur les poussées cyanobactériennes dans la retenue hypereutrophe de Grangent (France). Revue des sciences de l'eau / Journal of Water Science, 15(1), 289-299. https://doi.org/10.7202/705453ar

\section{Résumé de l'article}

Dans la retenue hypereutrophe de Grangent, le phytoplancton estival est dominé par la cyanobactérie Microcystis aeruginosa. Dans le but de lutter contre la formation de ces blooms cyanobactériens, une expérimentation de brassage artificiel épilimnique par aération a été réalisée en 1997-1998. Ce dispositif avait pour but de créer des turbulences supprimant l'avantage adaptatif que constitue, chez M. aeruginosa, la faculté de réguler sa flottabilité. Il devait également permettre l'homogénéisation des teneurs en oxygène dissous, la réduction des pics de $\mathrm{pH}$, de la turbidité des eaux superficielles et des teneurs en ammonium.

Les résultats escomptés ont été vérifiés pour les paramètres physicochimiques. Les valeurs se sont révélées plus homogènes, mais seulement à proximité des lignes de brassage et uniquement jusqu'à $10 \mathrm{~m}$ de profondeur. En revanche, les blooms cyanobactériens n'ont pas été réduits. Il apparait même au contraire que, sous l'influence du mélange, les cyanobactéries ont eu à leur disposition une plus grande quantité de nutriments qu'elles ont utilisés pour constituer des réserves glucidiques. Ainsi, en aval de la zone brassée, ces réserves ont permis une synthèse protéique plus importante.

Sur la retenue de Grangent, le dispositif de brassage peut offrir une solution palliative du point de vue touristique en limitant l'accumulation de cyanobactéries en surface, mais il ne permet pas d'éliminer, ni même de diminuer, les proliférations de M. aeruginosa en période estivale. 


\title{
Effet d'une expérimentation de brassage artificiel épilimnique par aération sur les poussées cyanobactériennes dans la retenue hypereutrophe de Grangent (France)
}

\author{
Impact of an epilimnic artificial mixing experiment \\ against growth of cyanobacteria in the hypertrophic \\ reservoir of Grangent (France)
}

In the reservoir of Grangent, a highly eutrophic lake located on the upper part of the Loire River, about 10 miles south of Saint-Étienne (France), Microcystis aeruginosa usually dominates the phytoplankton community in late summer and early autumn for many years. Mass developments of this cyanobacterium led to serious difficulties in multi-purpose usage. In order to fight against blooms, an epilimnic artificial mixing was experimented. $M$. aeruginosa is adapted to stable stratification of the water column. Therefore, partial destratification or bubbling with air are employed to replace $M$. aeruginosa by better grazable, non- «blooming » and non-toxic species. This cyanobacterium is supposed to lose its advantage of buoyancy and to reduce his growth. This system was also employed to reduce peaks of $\mathbf{p H}$, turbidity of surface waters and concentration of $\mathrm{NH}_{4}$ and to homogenize the dissolved oxygen concentration inside the water column.

Three lines of mixing were tested in 1998: one located at "Châtelet", upstream of reservoir, measuring $700 \mathrm{~m}$ at $11 \mathrm{~m}$ depth; one near the beach of Saint-Victor, with the same length and immersed to $15 \mathrm{~m}$ depth and, finally, a line of $\mathbf{4 0 0} \mathrm{m}$, near the port, at $16 \mathrm{~m}$ depth (figure 1).

Data were collected from representative sites, upstream, near and downstream the artificial mixing. They were sampled weekly since April to November 1998. At each site the vertical profiles of temperature and dissolved oxygen were measured (figure 2). For each sample, the parameters following were analyzed: $\mathrm{pH}, \mathrm{NO}_{3}, \mathrm{NH}_{4}, \mathrm{PO}_{4}$, carbohydrates, proteins, chlorophyll $a$ and phytoplankton enumeration.

Laboratoire de biologie animale et appliquée, Faculté des sciences et techniques, Université Jean-Monnet, 23, rue du Dr. P. Michelon, 42023 Saint-Etienne cedex 2, France.

* Correspondance. Email : delphine.latour@univ-st-etienne.fr

Les commentaires seront reçus jusqu'au 31 mars 2003. 
Concerning the physicochemical parameters, the assumptions were checked: the values appeared more homogeneous near the lines of mixing than at the other stations. For example, the average temperatures varied between $20,6^{\circ} \mathrm{C}$ (at $10 \mathrm{~m}$ depth) and $21,3^{\circ} \mathrm{C}$ (at $0,5 \mathrm{~m}$ depth) at Saint-Victor. This variation reached $1,3^{\circ} \mathrm{C}$ at the station Camaldules. On the other hand, this effect was perceived only up to $10 \mathrm{~m}$ of depth and at a limited distance of mixing.

In the same way, the colonies of $M$. aeruginosa were mixed in the water column but only up $10 \mathrm{~m}$ depth and near mixing. Moreover, their growth has not decreased on the whole of reservoir. In period of bloom (August 25), G/P ratio was higher in the mixing zone than in the neighbourhoods, primarily because of an increase in carbohydrates (figure 3 ). In the mixed zone, $M$. aeruginosa seemed to benefit greater quantity of mineral elements it could use to constitute carbohydrates reserves (figure 4). In this way, when the conditions that became less favourable, like downstream, cyanobacteria were able to follow their development by synthesizing proteins starting from their reserves in carbohydrates.

In the reservoir of Grangent, artificial mixing did not allow to fight effectively against blooms of cyanobacteria. Colonies of $M$. aeruginosa were simply diluted in the water column near mixing but did not reduce their growth.

Key-words: artificial mixing, Microcystis aeruginosa, cyanobacteria, carbohydrates, proteins, eutrophic reservoir.

Dans la retenue hypereutrophe de Grangent, le phytoplancton estival est dominé par la cyanobactérie Microcystis aeruginosa. Dans le but de lutter contre la formation de ces blooms cyanobactériens, une expérimentation de brassage artificiel épilimnique par aération a été réalisée en 1997-1998. Ce dispositif avait pour but de créer des turbulences supprimant l'avantage adaptatif que constitue, chez $M$. aeruginosa, la faculté de réguler sa flottabilité. Il devait également permettre l'homogénéisation des teneurs en oxygène dissous, la réduction des pics de $\mathrm{pH}$, de la turbidité des eaux superficielles et des teneurs en ammonium.

Les résultats escomptés ont été vérifiés pour les paramètres physicochimiques. Les valeurs se sont révélées plus homogènes, mais seulement à proximité des lignes de brassage et uniquement jusqu'à $10 \mathrm{~m}$ de profondeur. En revanche, les blooms cyanobactériens n'ont pas été réduits. Il apparaît même au contraire que, sous l'influence du mélange, les cyanobactéries ont eu à leur disposition une plus grande quantité de nutriments qu'elles ont utilisés pour constituer des réserves glucidiques. Ainsi, en aval de la zone brassée, ces réserves ont permis une synthèse protéique plus importante.

Sur la retenue de Grangent, le dispositif de brassage peut offrir une solution palliative du point de vue touristique en limitant l'accumulation de cyanobactéries en surface, mais il ne permet pas d'éliminer, ni même de diminuer, les proliférations de $M$. aeruginosa en période estivale.

Mots clés : brassage artificiel, Microcystis aeruginosa, cyanobactéries, glucides, protéines, retenue eutrophe. 


\section{1 - INTRODUCTION}

La retenue de Grangent est le siège d'une forte production phytoplanctonique (diatomées et chlorophycées) et chaque fin d'été des fleurs d'eau cyanobactériennnes à Microcystis aeruginosa se développent depuis plusieurs années (BERTHON et al., 1996). La prolifération de cette cyanobactérie engendre des nuisances d'ordre touristique, une menace pour la survie piscicole en réduisant les teneurs en oxygène et un danger pour la santé publique lié à la libération de toxines dans l'eau.

Afin d'améliorer la qualité de l'eau de la retenue, un Plan d'action renforcé (PAR) a été mis en place en 1990 par le Conseil général de la Loire. Ce dernier avait pour objectif de mieux traiter les rejets des communes riveraines de façon à réduire les apports en phosphore responsables de l'eutrophisation. En complément de cette démarche dont les effets ont commencé à se faire sentir (SALENÇON, 1997), une action palliative a été engagée afin d'obtenir rapidement une diminution des fleurs d'eau cyanobactériennes au niveau de la zone touristique de la retenue, à la station de Saint-Victor. Des lignes de brassage artificiel ont été implantées en amont et en aval de ce site dans le but de destratifier partiellement et localement la retenue et ainsi limiter la croissance des cyanobactéries (KÖHLER, 1992 ; VISSER et al., 1996).

En effet, $M$. aeruginosa est adaptée à une stratification marquée de la colonne d'eau (SHAPIRO, 1973). De plus, en conditions stables, cette cyanobactérie est capable de réguler sa flottabilité par l'intermédiaire de vésicules gazeuses. Cet avantage adaptatif lui permet de recevoir une plus forte dose journalière de lumière que le reste du phytoplancton soumis à la sédimentation. En revanche, en situation de brassage artificiel, les colonies de $M$. aeruginosa sont en mouvement permanent dans la colonne d'eau et perçoivent globalement moins de lumière que celles qui s'agglomèrent à la surface lors d'une fleur d'eau. Elles réduisent alors leur multiplication (VISSER et al., 1996).

Outre le fait de lutter contre le développement estival des cyanobactéries, le dispositif de brassage artificiel installé en 1997-1998 sur la retenue de Grangent devait également permettre l'homogénéisation des teneurs en oxygène dissous, la réduction des pics de $\mathrm{pH}$, de la turbidité des eaux superficielles et des teneurs moyennes en $\mathrm{NH}_{4}$ (Aquatechnique, 1999).

Afin de connaître l'impact réel de ce procédé de brassage sur les fleurs d'eau à $M$. aeruginosa, un suivi scientifique de la qualité de l'eau a été réalisé en 1997 et 1998.

\section{2 - MATÉRIEL ET MÉTHODES}

\subsection{Présentation du site}

La retenue de Grangent, mise en eau en 1957, est située sur le cours amont de la Loire. La superficie du plan d'eau est de 365 ha avec une longueur de 
$21 \mathrm{~km}$, une largeur maximale de $400 \mathrm{~m}$, une profondeur maximale de $50 \mathrm{~m}$ au droit du barrage et une capacité de 57,4 millions de $\mathrm{m}^{3}$.

La fonction principale de cet ouvrage est la production d'électricité mais il joue également un rôle important dans l'irrigation de la plaine du Forez via un canal. Avec la base nautique de Saint-Victor, la retenue développe aussi une activité touristique importante.

\subsection{Dispositif de brassage}

Les lignes de bullage ont été implantées dans une zone où l'activité touristique se trouve concentrée, c'est-à-dire à proximité de la base nautique de Saint-Victor.

Le brassage est assuré par un réseau de canalisations immergé, diffusant de l'air sous forme de rideaux de bulles (figure 1).

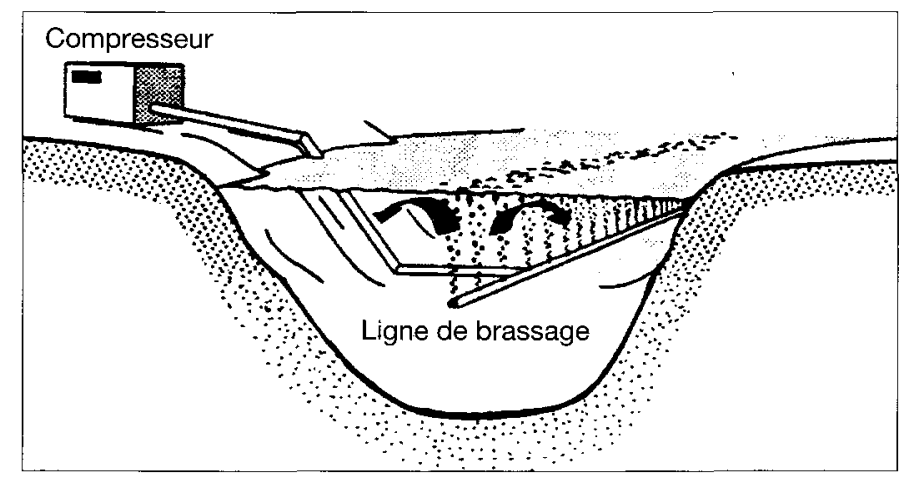

Figure 1 Dispositif du brassage artificiel de l'épilimnion.

Artificial mixing system of epilimnion.

Trois lignes de brassage ont fonctionné durant l'année 1998 : une située au Châtelet, en amont de la retenue, mesurant $700 \mathrm{~m}$ et se situant à $11 \mathrm{~m}$ de profondeur ; une à proximité de la plage de Saint-Victor, de même longueur et immergée à $15 \mathrm{~m}$ de fond, et enfin une ligne de $400 \mathrm{~m}$, au droit du port, disposée à $16 \mathrm{~m}$ de profondeur (figure 2).

Les lignes de brassage étaient alimentées par un compresseur situé sur les berges au bout de la plage de Saint-Victor (figure 2).

\subsection{Suivis physicochimique et biologique}

Les paramètres physicochimiques mesurés ont été choisis afin de vérifier les engagements de la société Aquatechnique concernant l'homogénéisation sur toute la colonne d'eau de certains d'entre eux. Les analyses biologiques ont été réalisées dans le but de préciser l'impact réel du brassage sur la répartition et l'état physiologique du phytoplancton.

Des échantillons ont été récoltés en amont de la retenue (station Révotes), dans la zone touristique à une distance de $25 \mathrm{~m}$ du brassage (station Saint-Victor) et en aval (station Camaldules) à l'aide d'une bouteille de type Van Dorn 


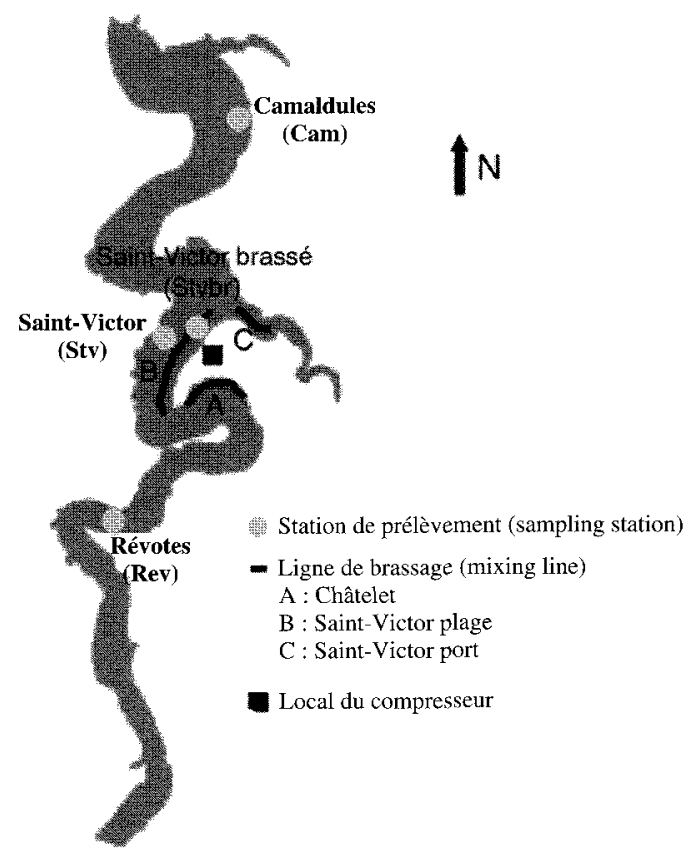

Figure 2 Localisation des lignes de brassage artificiel sur la retenue de Grangent.

Place of the artificial mixing lines in the reservoir of Grangent.

tous les $2,5 \mathrm{~m}$ de profondeur sur toute la colonne d'eau et suivant un pas hebdomadaire, du mois d'avril au mois de novembre 1998 (figure 2).

À chaque station, les profils verticaux de température et d'oxygène ont été relevés à l'aide d'une sonde. La transparence de l'eau a été mesurée in situ à l'aide d'un disque de Secchi.

Pour chaque échantillon, les paramètres suivant ont été analysés : $\mathrm{pH}, \mathrm{NO}_{3}$, $\mathrm{NH}_{4}, \mathrm{PO}_{4}$, glucides (MONTREUIL et SPICK, 1963), protéines (LOWRY et al., 1951), chlorophylle a (LORENZEN, 1967) et dénombrement phytoplanctonique sous microscope inversé selon la méthode d'UTERMÖHL (1958) modifiée par LEGENDRE et WATT $(1971,1972)$. De plus, les cinq derniers paramètres biotiques cités ont été étudiés à une station supplémentaire située dans la colonne d'eau brassée, au niveau du rideau de bulles d'air : il s'agit de la station « Saint-Victor brassé » (STV br) (figure 2).

\section{3 - RÉSULTATS}

L'année 1998 a constitué une année atypique ponctuée de plusieurs périodes de crues et de mauvais temps pendant la première moitié de l'été. Ceci s'est répercuté sur les équilibres physicochimiques et les successions phytoplanctoniques. 


\subsection{Impact du brassage sur les paramètres physicochimiques et sur la biomasse phytoplanctonique}

Depuis la mise en fonctionnement du dispositif de brassage, les profils verticaux de température à la station de Saint-Victor sont apparus plus homogènes entre 0 et $10 \mathrm{~m}$ de profondeur qu'aux autres stations. En effet, en période estivale, les températures moyennes ont varié de $20,6^{\circ} \mathrm{C}$ (à $10 \mathrm{~m}$ ) à $21,3{ }^{\circ} \mathrm{C}(\mathbf{a} 0,5 \mathrm{~m})$, soit un écart de $0,7^{\circ} \mathrm{C}$ à Saint-Victor, alors que cet écart a été de $1,3^{\circ} \mathrm{C}$ à la station Camaldules.

De même, les profils d'oxygène dissous ont été plus homogènes à SaintVictor qu'à la station de Camaldules (figure 3). L'oxycline était installée autour de $15 \mathrm{~m}$ à Saint-Victor alors qu'elle était située 2 à $4 \mathrm{~m}$ moins profond à la station Révotes. En revanche, la teneur en oxygène a été plus faible en surface à Saint-Victor.

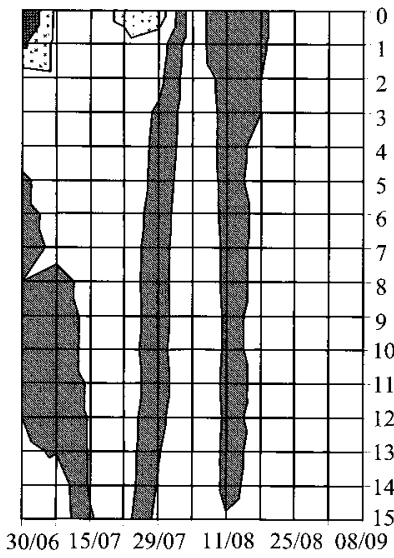

Date

Saint-Victor

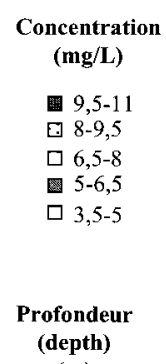

(m)

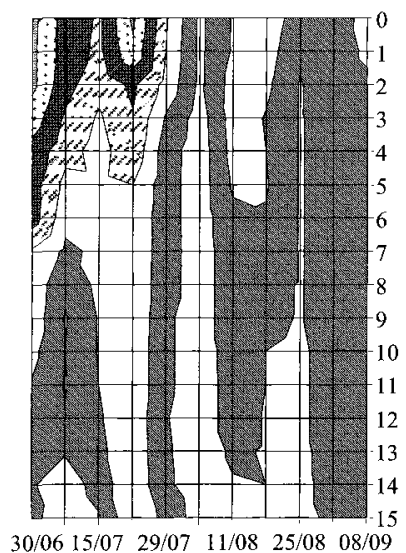

Date

Camaldules

Figure 3 Évolution spatiotemporelle des teneurs en oxygène dissous. Spatio-temporal evolution of dissolved oxygen content.

Le brassage de la colonne d'eau 0-10 $\mathrm{m}$ a influé sur les valeurs de $\mathrm{pH}$, qui ont été inférieures à $25 \mathrm{~m}$ des lignes d'aération : elles ont été comprises entre 7 et 8,5 à Saint-Victor alors qu'elles ont fluctué de 7,5 à 9,25 aux autres stations.

De façon similaire, la turbidité de l'eau, estimée à partir de la profondeur de disparition du disque de Secchi, a été légèrement plus faible à Saint-Victor (entre 1,5 et $3 \mathrm{~m}$ ) qu'aux autres stations (entre 1,3 et 2,3 m), certainement en relation avec la dilution de la biomasse algale à cette station. En effet, les teneurs en chlorophylle a sont plus homogènes à Saint-Victor, fluctuant au cours de la saison entre 20 et $60 \mu \mathrm{g} \cdot \mathrm{L}^{-1}$, qu'aux autres stations où elles ont varié entre 10 et $80 \mu \mathrm{g} \cdot \mathrm{L}^{-1}$. De plus, ces pigments ont affiché des pics moins importants à proximité du bullage.

Les dénombrements phytoplanctoniques confirment ces observations. Les chlorophycées se sont développées simultanément sur l'ensemble de la retenue avec un pic principal fin juillet. Toutefois, le brassage artificiel a conduit à 
une dilution verticale à Saint-Victor où la concentration en chlorophycées a été comprise entre 1000 et $1500 \mathrm{cell} \cdot \mathrm{L}^{-1}$, contre 2000 à $2500 \mathrm{cell} \cdot \mathrm{L}^{-1}$ aux Révotes. La biomasse des cyanobactéries, essentiellement composée de Microcystis aeruginosa, s'est globalement accrue d'amont en aval avec un développement maximal fin août. Le brassage artificiel a mené ici à une répartition différente des colonies cyanobactériennes dans la masse d'eau : ces dernières ont été dispersées entre 0 et $9 \mathrm{~m}$ à Saint-Victor alors qu'elles sont restées localisées en surface aux Camaldules (entre 0 et $4 \mathrm{~m}$ ). En revanche, la répartition des diatomées n'a pas été perturbée par le brassage artificiel puisque leur principal pic de développement a eu lieu fin juin alors que les installations n'étaient pas encore en service.

Concernant les teneurs en éléments nutritifs, le système de brassage n'a pas eu d'impact sur les concentrations en phosphore et en ammonium puisque celles-ci sont identiques à toutes les stations. Par ailleurs, les lignes de bullage étant disposées à $20 \mathrm{~m}$ du fond, le phénomène de relargage du phosphore par le sédiment ne se trouve pas amplifié sous l'effet du bullage.

En revanche, un léger effet a été mis en évidence pour les teneurs en nitrates dont les valeurs ont été plus homogènes à Saint-Victor (de 2,9 à $4,1 \mathrm{mg} \cdot \mathrm{L}^{-1}$ ) qu'aux Révotes (de 1,1 et $4,9 \mathrm{mg} \cdot \mathrm{L}^{-1}$ ).

\subsection{Impact du brassage sur l'état physiologique du phytoplancton}

Au cours de la saison, la limite inférieure de la zone euphotique à Saint-Victor était comprise entre $3 \mathrm{~m}$ et $7,5 \mathrm{~m}$. Les lignes de bullage étant implantées à une profondeur de $15 \mathrm{~m}$, l'ensemble de cette zone et donc le phytoplancton ont été mélangés.

Afin de vérifier l'efficacité du système, une étude comparative de l'état physiologique du phytoplancton a été menée. Pour cela, les teneurs en glucides et en protéines ainsi que les dénombrements cellulaires ont été comparés aux stations suivantes : Révotes, Saint-Victor et Camaldules (figure 2).

Avant l'apparition des cyanobactéries, le 21 juillet 1998, le rapport glucides/protéines (G/P) à la station Saint-Victor était égal ou supérieur à celui des autres stations. Après cette date, il est inférieur en cette station (figure 4). Cette chute du rapport $G / P$ était essentiellement liée à la diminution des teneurs en glucides. Il apparaît donc que le phytoplancton, dominé alors par des colonies de $M$. aeruginosa (renfermant environ $50 \cdot 10^{3}$ cellules), accumulerait moins de réserves sous forme glucidique à Saint-Victor qu'aux autres stations.

Pour vérifier si cette différence d'activité métabolique était liée à la présence proche du dispositif de brassage, une comparaison du rapport G/P a été effectuée en période de bloom à $M$. aeruginosa (25 août) entre la station de Saint-Victor à $25 \mathrm{~m}$ de la ligne de bullage (Stv), et une autre station située sur la ligne de brassage (Stv br). Les résultats ont indiqué un rapport G/P faible à Stv $(=8)$ alors qu'il était très élevé à Stv br $(=30)$. Cette différence notable s'explique par des teneurs en glucides beaucoup plus importantes à l'intérieur même de la zone brassée (Stv br) qu'à une distance de 25 m (Stv) (figure 5). Dans la zone brassée, les cyanobactéries auraient donc une forte activité anabolique et constitueraient des réserves glucidiques importantes. En revanche, à une distance de $25 \mathrm{~m}$ du brassage (Stv) les cyanobactéries se trouveraient dans une situation moins favorable et utiliseraient alors massivement leur réserves glucidiques. 
$\mathrm{G} / \mathrm{P}$

$10^{6} \mathrm{cell} . / \mathrm{L}$

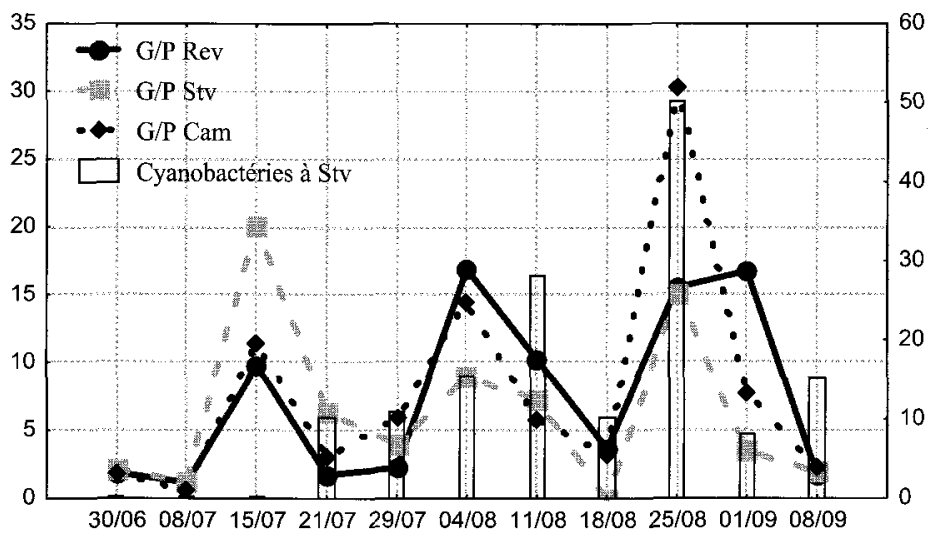

Figure 4 Évolution spatiotemporelle du rapport G/P.

Spatio-temporal evolution of G/P ratio.

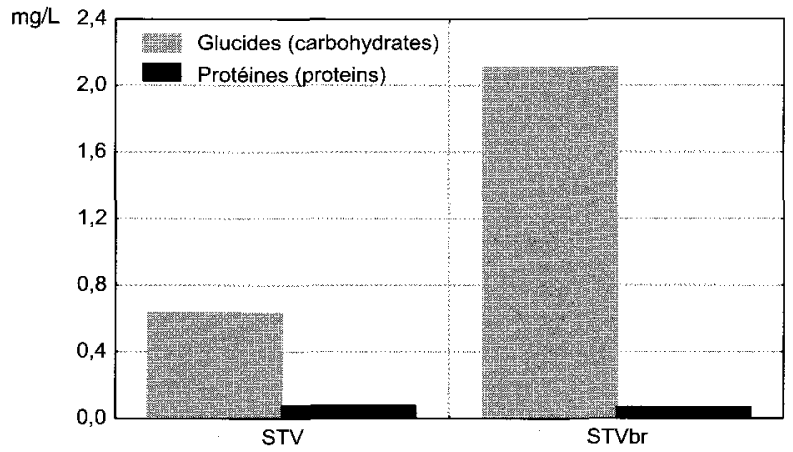

Figure 5 Teneurs en glucides et protéines en période de bloom à Microcystis.

Contents of carbohydrates and proteins in period of bloom with Microcystis.

\section{4 - DISCUSSION ET CONCLUSION}

Déjà en 1974, DUNST et al. avaient dénombré 123 lacs utilisant des systèmes d'aération artificielle pour améliorer la qualité de l'eau.

D'un point de vue physicochimique, de nombreux travaux ont montré, comme sur la retenue de Grangent, qu'un brassage artificiel homogénéise sur. toute la colonne d'eau les teneurs en oxygène, le $\mathrm{pH}$, la température ou encore les concentrations en nitrates (STEINBERG et ZIMMERMANN, 1988 ; VISSER et al;, 1996). 
En revanche, la profondeur de la retenue de Grangent, plus importante que celle d'autres lacs soumis à un brassage artificiel $(45 \mathrm{~m}$ de profondeur à la station Saint-Victor contre $18 \mathrm{~m}$ de profondeur moyenne sur le lac Nieuwe Meer et $11 \mathrm{~m}$ pour le lac Fischkaltersee), explique dans notre cas la limitation de l'impact du système de bullage.

Même si le nombre d'installations de brassage artificiel ne cesse de croître, l'efficacité de tels dispositifs reste très controversée, notamment pour la lutte contre les blooms cyanobactériens. II est vrai que les colonies de $M$. aeruginosa sont diluées dans la masse d'eau et ne forment plus de plaques à la surface de la zone brassée, mais, à l'échelle de la retenue, la croissance cyanobactérienne ne semble pas être altérée. Au contraire, dans la zone brassée, l'augmentation des teneurs en glucides traduit une mise en réserve énergétique.

TOETZ (1981) a démontré que si la profondeur de mélange est en dessous du point de compensation photique, il se produit vers la surface un apport de nutriments qui étaient auparavant transférés dans l'hypolimnion. Par l'intermédiaire du brassage, les cyanobactéries auraient donc à leur disposition une plus grande quantité d'éléments minéraux leur permettant de constituer un pool glucidique utilisable dans des conditions moins favorables.

D'après VISSER et al. (1996), le taux de croissance de $M$. aeruginosa est plus élevé dans les secteurs où le brassage est inefficace. En effet, les réserves glucidiques engrangées sont utilisées pour le développement des cyanobactéries et servent, entre autres, à la synthèse protéique. Ce processus est directement observable en aval, où les teneurs en protéines augmentent de $0,08 \mathrm{mg} \cdot \mathrm{L}^{-1}$ à Saint-Victor à $1,8 \mathrm{mg} \cdot \mathrm{L}^{-1}$ à la station Camaldules.

D'autres travaux ont montré qu'un dispositif de brassage artificiel ne suffit pas pour faire disparaître les cyanobactéries des successions phytoplanctoniques. Même si la dominance des diatomées peut souvent être prolongée sous l'action d'un brassage artificiel, la prolifération des cyanobactéries est inévitable dès que la silice devient limitante (HAWKINS et GRIFFITHS, 1993). De même, sur le lac East Sidney, la taille des populations de cyanobactéries n'a pas été réduite par une déstratification artificielle (BARBIERO et al., 1996). Une étude réalisée sur 50 lacs en Australie a montré que le succès de la déstratification artificielle est limité (MCAULIFFE et ROSICH, 1990). Seulement $24 \%$ des cas affichaient un franc succès contre $28 \%$ d'échec, $46 \%$ avec un impact limité et $2 \%$ sans résultat. Les systèmes de brassage s'avèrent être performants pour les problèmes associés à la couleur de l'eau et à la turbidité. En revanche, pour le contrôle du développement phytoplanctonique, ce processus se révèle nettement moins approprié.

Cependant, il existe dans la littérature certaines expérimentations démontrant l'efficacité d'un brassage artificiel contre les proliférations cyanobactériennes et plus globalement contre l'augmentation de la biomasse phytoplanctonique. Une augmentation du rapport $Z_{\mathrm{m}} 1 / Z_{\mathrm{eu}}{ }^{2}$ diminue le temps passé dans la zone euphotique, entraînant la réduction de la production planctonique par photosynthèse et par conséquent sa croissance (FAST, 1981 ; LORENZEN et MITCHELL, 1975). Ainsi,

1. Profondeur mélangée.

2. Profondeur euphotique. 
STEINBERG et ZIMMERMANN (1988) ont utilisé avec succès un brassage intermittent pour réduire la dominance de cyanobactéries (surtout des Oscillaires) et diminuer la biomasse phytoplanctonique en général. Plus proche encore de notre étude, sur le Blelhalm Tarn, REYNOLDS et al. (1984) ont observé la réduction de la croissance de Microcystis.

Le brassage artificiel des eaux de la retenue de Grangent n'a pas permis d'éliminer les blooms estivaux de $M$, aeruginosa, sans doute à cause de sa trop faible portée à l'échelle du plan d'eau. Une campagne de marquage des masses d'eau à la fluorescéine a permis de déterminer l'ampleur exacte de la zone influencée par le dispositif de brassage. De part et d'autre des lignes de bullage, il se forme une cellule convective dont la profondeur est égale à la hauteur d'immersion des lignes (de $11 \mathrm{~m}$ à $16 \mathrm{~m}$ suivant les sites) et la largeur avoisinant $25 \mathrm{~m}$ (Aquatechnique, 1999). Ceci a conduit à estimer un flux d'échange par mètre linéaire de $126 \mathrm{~m}^{3} \cdot \mathrm{h}^{-1}$ pour la ligne localisée à Saint-Victor. À proximité des lignes de bullage, les colonies de cyanobactéries ont donc simplement été homogénéisées sur une profondeur d'environ $10 \mathrm{~m}$ comme un grand nombre de paramètres physicochimiques. Ces résultats peuvent être en partie attribués à la trop petite surface brassée par rapport à la surface totale de la retenue. En effet, même si les cellules de convection engendrées par le brassage se propagent à plusieurs dizaines de mètres des lignes de bullage, cela ne représente qu'une faible proportion de la surface totale du lac.

VISSER et al. (1996) considèrent que $80 \%$ du volume d'eau doit subir le mélange pour obtenir des résultats satisfaisants. C'est loin d'être le cas dans la retenue de Grangent dont la plus grande partie n'a pas été touchée par le système de brassage artificiel. Ce dernier constitue donc uniquement une solution palliative du point de vue touristique en limitant la formation de plaques cyanobactériennes aux abords de la plage.

\section{RÉFÉRENCES BIBLIOGRAPHIQUES}

AQUATECHNIQUE, 1999. Impact des lignes de brassage sur le fonctionnement du lac de Grangent. Rapport provisoire, $73 \mathrm{p}$.

BARBIERO R.P., SPEZIALE B.J., ASHBY S.L., 1996. Phytoplankton community succession in a lake subjected to artificial circulation. Hydrobiologia, 331, 109120.

BERTHON J.-L., DEVAUX J., ALEYA L., GIRAUDET H., RESTITUITO F., 1996. Déterminisme de l'eutrophisation de la retenue de Grangent (Loire) : étude des nutriments, de la dynamique des populations phytoplanctoniques et des relations phytozooplanctoniques en 1990-1991. Hydroécol. Appl., 8, 99-125.

DUNST R.C., BORN S.M., UTTOMARK P.D., SMITH S.A., NICKOLS S.A., PETERSON J.O., KNAUER D.R., SERNS S.L., WIN-
TER D.R., WIRT T.I., 1974. Survey of lake the rehabilitation techniques and experiences. Dep. Nat. Resour., Madison. Tech. Bull., 75, 1-179.

FAST A.W., 1981. The effects of artificial destratification on algae populations. In: POWLING F. L., I. J. (Ed.), Destratification of lakes and reservoirs to improve Water Quality Burns, Aust. Government Publ., Camberra,515-556.

HAWKINS P.R., GRIFFITHS D.J., 1993. Artificial destratification of small tropical reservoir: effects upon the phytoplankton. Hydrobiologia, 254, 169-181.

KÖHLER J., 1992. Influence of turbulent mixing on growth and primary production of Microcystis aeruginosa in the hypertrophic Bautzen Reservoir. Arch. Hydrobiol., 123, 413-429. 
LEGENDRE L., WATT W.D., 1971-1972. On rapid technique for enumeration. Ann. Inst. Oceanogr., Paris, 58, 173-177.

LORENZEN C.J., 1967. Determination of chlorophyll and pheopigments: spectrophotometric equation. Limnol. Oceanogr., 12, 343-346.

LORENZEN M.W., MITCHELL R., 1975. An evaluation of artificial destratification for control of algal blooms. J. Amer. Water Works Ass., 67, 373-377.

LOWRY O.H., ROSEBROUGH N.J., LEWIS A., RANDALL R.J., 1951. Protein measurement with the Folin phenol reagent. J. Biol. Chem., 193, 265-275.

MCAULIFFE T.F., ROSICH R.S., 1990. The triumphs and tribulations of artificial mixing in australian water bodies. Water, 22-23.

MONTREUIL J., SPICK G., 1963. Microdosage des glucides. Méthodes colorimétriques de dosage des glucides totaux. Monographie du Laboratoire de Chimie Biologique. Fac. Sci. Lille, t.1, 131 p.

REYNOLDS C.S., WISEMAN S.W., CLARKE M.J.O., 1984. Growth and loss-rate responses of phytoplankton to intermittent artificial mixing and their potential application to the control of planktonic algal biomass. J. Appl. Ecol., 21, 11-39.

SALENCON M.J., 1997. Évolution de l'écosystème de la retenue de Grangent en 1996. Comparaison aux années précédentes. Rapport EDF HE/3197/028, Electricité de France, Paris, $44 \mathrm{p}$.

SHAPIRO J., 1973. Blue-green algae: why they become dominant. Science, 179, 382-384.

STEINBERG C., ZIMMERMANN G.M., 1988. Intermittent destratification: a therapy measure against cyanobacteria in lakes. Environ. Technol. Letters, 9, 337-350.

TOETZ D.W., 1981. Effects of whole lake mixing on water quality and phytoplankton. Water Res., 15, 1205-1210.

UTERMOHL H., 1958. Zur Vervollkommung der quantitative Phytoplankton-Methoik. Mitt. Inter. Ver. Limnol., 9, 1-38.

VISSER P.M., IBELINGS B.W., VAN DER VEER B., KOEDOODS J., MUR L.R., 1996. Artificial mixing prevents nuisance blooms of the cyanobacterium Microcystis in lake Nieuwe Meer, the Netherlands. Freshwater Biol., 36, 435-450. 